\title{
MODELOS DE MATURIDADE DE TESTE DE SOFTWARE: UMA REVISÃO SISTEMÁTICA DA LITERATURA
}

\section{Software Test Maturity Models: A Systematic Review of the Literature}

\section{Juliano Rodrigues Ramos ${ }^{1}$}

\author{
${ }^{1}$ Universidade do Oeste Paulista - Unoeste \\ Graduado em Sistemas de Informação pela Faculdade de Informática de Presidente \\ Prudente - FIPP \\ Presidente Prudente - São Paulo, Brasil. \\ juliano_rr@yahoo.com.br
}

RESUMO - O alto nível de abstração dos modelos de melhoria de processos de software e a importância da qualidade dos produtos de software fazem com que os termos Maturidade de Teste (TMA) e Melhoria do Processo de Teste (TPI) ganhem importância na indústria e nas pesquisas de Software. O objetivo deste trabalho é delinear, a partir de uma revisão de literatura, resultados adicionais aos trabalhos existentes sobre modelos de maturidade de Processos de Teste (TMMi, TPI(Next) e MPT.br). Foi feita uma revisão sistemática da literatura com base de dados disponíveis na Web. Os resultados mostram uma síntese dos três modelos de maturidade de teste pesquisados, considerações referentes ao estudo destes modelos na academia e comparações gráficas das características desses modelos. Conclui-se que há muitos modelos de maturidade de teste propostos na literatura, sendo o TMMi e o TPI os mais utilizados a nível internacional, e o MPT.br o modelo no contexto brasileiro.

Palavras-chave: processo de software; teste de software; Avaliação de Maturidade de Teste (TMA); Melhoria do Processo de Teste (TPI).

ABSTRACT - The high level of abstraction of software process improvement models and most software products make Maturity Test (TMA) and Test Process Improvement (TPI) win in industry and software research. The objective of this work is to delineate, from a literature review, the results are compared with the maturity models of Testing Processes (TMMi, TPI (Next) and MPT.br). The systemic basis of literature based on data available on the web. The results are a synthesis of the three types of test maturity investigated, being that they are part of the academic model and the comparisons are characteristic of the models. In conclusion there are many maturity models of tests proposed in the literature, with TMMi and TPI being the

Recebido em: 04/06/2018 Revisado em: 12/09/2018 Aprovado em: 18/09/2018 most usable at an international level, and MPT.br the model in the Brazilian context.

Keywords: software process; software testing; Test Maturity Assessment (TMA); Testing Process Improvement (TPI). 


\section{INTRODUÇÃO}

A Validação e Verificação são duas atividades que as empresas de desenvolvimento de software devem obrigatoriamente executar para gerar produtos com alto padrão de qualidade (MONTEIRO; MACHADO; KAZMAN, 2009). As abordagens sobre a melhoria do processo de teste de software têm a função de conduzir as organizações que desenvolvem softwares, no intuito de melhorar o processo de teste dessas entidades (AFZAL et al., 2016).

Há, ainda, um grande interesse por parte das empresas em avaliar e melhorar a maturidade de suas práticas e processos de teste de software (GAROUSI; FELDERER; HACALOĞLU, 2017), pois, em muitas companhias, essas práticas ainda são dependentes de consultorias ad-hoc (GAROUSI et al., 2015; GAROUSI; ZHI, 2013; GRINDAL; OFFUTT; MELLIN, 2006). Um estudo da Universidade de Cambridge, em 2013, mostra que o custo anual com a detecção e ajustes de problemas em softwares é de aproximadamente 312 bilhões de dólares, e que tal tarefa corresponde à metade (50\%) do tempo médio de desenvolvimento do projeto (BRITTON et al., 2013).

O objetivo desse trabalho é identificar os modelos de maturidade de testes propostos para a melhoria do processo de teste de software. A principal contribuição desse trabalho é disponibilizar um levantamento dos principais trabalhos científicos com uma abordagem de revisão de literatura sobre a Avaliação de Maturidade de Teste (TMA - Test Maturity Assessment) e a Melhoria de Processo de Teste (TPI - Test Process Improvement) para identificar pontos incrementais aos estudos já existentes.

\section{MODELOS DE MATURIDADE DE PROCESSO DE TESTE}

Na última década, a indústria de desenvolvimento de software intensificou a atenção ao tema Qualidade de Software. A abordagem de modelos de maturidade - de Crosby, Philip (1926-2001) - vem sendo adotada como modelos de melhoria de processos em grande parte da indústria.

Os modelos gerais de maturidade de processo de software amplamente utilizados são o CMMi e o MPS.br, no âmbito nacional. Divididos em cinco níveis de maturidade, e sete para o caso do MPS.br, ambos possuem a abordagem da qualidade espalhada em 2 níveis distintos: "Garantia da Qualidade" e "Verificação e Validação" (GIS-MPSF, 2016; GD-CMMI, 2010). Algumas empresas, no entanto, são resistentes a adoção do nível 2 do CMM ou $\mathrm{F}$ para o nível correspondente MPS.br, porque não consideram esse nível de maturidade um benefício, já que sua implementação é dispendiosa financeiramente e não cobre os esforços de validação e de verificação (MONTEIRO; MACHADO; KAZMAN, 2009).

Ainda que os modelos gerais de melhoria de processo, CMMi e MPS.br, possuam requisitos de qualidade, verificação e validação, estes possuem uma abordagem em um alto nível de abstração, o que fez com que especialistas e pesquisadores da área de testes criassem modelos específicos para o setor, dando origem a modelos como o TMM, TPI e MPT.br (SILVA; CHAIM, 2017). Várias abordagens têm sido propostas para conduzir o processo de Avaliação de Maturidade de Teste (TMA - Test Maturity Assessment) e Melhoria de Processo de Teste (TPI - Test Process Improvement) (GAROUSI; FELDERER; HACALOĞLU, 2017). Um esquema geral do processo de TMA/PPI é apresentado em Garousi, Felderer e Hacaloğlu (2018), onde descrevem que a iniciativa de TMA e TPI se inicia quando um engenheiro de teste ou uma equipe de teste determinam as iniciativas necessárias. Na sequência, há a promoção da conscientização entre as partes interessadas e a gestão. O engenheiro ou a equipe responsável determina em seguida as áreas de consideração, o modelo de TMA e TPI a serem utilizados e a abordagem adequada. Depois disso, o TMA real é 
iniciado, identificando as áreas de TPI. Na sequência, o engenheiro ou equipe planejam e conduzem o TPI e avaliam seus resultados e benefícios. Se mais melhorias (TPI) são necessárias, o processo se repete em um ciclo contínuo até o término esperado (GAROUSI; FELDERER; HACALOĞLU, 2018).

\subsection{TMMi}

O TMMi é baseado no TMM (Testing Maturity Model - Modelo de Maturidade de Teste), que por sua vez, é fundamentado no Modelo de Maturidade da Capacidade (CMC) e no CMMi (Garousi et al., 2017). A última versão da certificação TMMi é a 1.0 (TMMi FOUNDATION, 2018). A Fundação TMMi é a responsável por preparar e publicar as versões do TMMi. Trata-se de uma organização sem fins lucrativos, com sede na Irlanda, que apoia empresas no mundo todo visando um aumento na qualidade $e$ eficiência do software que as empresas desenvolvem (TMMi FOUNDATION, 2018).

Em uma avaliação TMMi, mede-se a maturidade dos processos de teste, e podese, ainda, determinar se a empresa atingiu determinado nível de maturidade de teste ou não (VEENENDAAL; WELLS, 2012).

Há um total de cinco níveis de maturidade no TMMi, cada nível de maturidade tem várias áreas de processo (VEENENDAAL; WELLS, 2012), e cada área tem metas e práticas específicas e genéricas (NETO et al., 2013).

Uma organização avança nesses diferentes níveis à medida que seu processo de teste evolui de um, que é ad-hoc e não gerenciado, para um que é gerenciado, definido, medido e otimizado. Assim, ao atingir cada nível, garante-se que todas as metas do mesmo foram alcançadas e as melhorias formaram a base para o próximo nível (VEENENDAAL; WELLS, 2012).

\subsection{TPI}

O TPI (Test Process Improvement Melhoria de Processo de Teste) é uma metodologia proposta por Koomen e Pol, em 1997, com o objetivo de permitir que as organizações avaliem a maturidade de seus processos de teste (VISSER et al., 2013). A arquitetura do TPI é constituída por 20 áreas chave, as quais são agrupadas em quatros níveis de maturidade, quais sejam: inicial, controlado, eficiente e otimizado (VISSER et al., 2013). Em cada um desses níveis, pontos de verificação (checkpoints) são utilizados para verificar a maturidade da organização em cada nível (VISSER et al., 2013). Um total de 300 pontos de verificação são utilizados no TPI (AFZAL et al., 2016). Portanto, se um processo de teste passa em todos os pontos de verificação de um determinado nível, o processo de teste é, então, classificado nesse nível. Uma Matriz de Maturidade de Teste fornece uma visão geral da maturidade de teste da organização avaliada, por destacar os pontos de verificação satisfeitos e os níveis de maturidade por área chave (AFZAL et al., 2016; GARCíA et al., 2018).

O sucessor do TPI é o TPI Next, o qual foi desenvolvido pela companhia Alemã Sogeti (AFZAL et al., 2016). O número de áreas chave no TPI Next foi reduzido para 16, e o número de pontos de verificação para 157 (AFZAL et al., 2016). Porém, outros elementos foram introduzidos nesse novo modelo para tratar das necessidades das organizações mais eficientemente no que tange à Melhoria de Processos de Teste.

\subsection{MPT.br}

O MPT.Br (Melhoria do Processo de Teste Brasileiro) é um modelo criado em 2010, a partir de uma parceria entre a SoftexRecife e a Riosoft, com o apoio do SEBRAE (MPT, 2018). O foco desse modelo é auxiliar empresas de pequeno e médio porte, desenvolvedoras de software, a melhorar seus processos de teste de software (MPT, 2018). A base do MPT.Br são outros modelos de referência em teste de software e de referência em melhoria de processo de software, tais como o TMM, criado por David Gelperin, o TPI, o CMMI (Capability Maturity Model Integration), criado pelo Instituto de Engenharia de software, e o MPS.BR (Melhoria de Processo do Software 
Brasileiro), criado em 2003 pela Sociedade SOFTEX (MPT, 2018).

Assim como o TMMi, o MPT.Br é composto por cinco níveis de maturidade, que são: parcialmente gerenciado (nível 1), gerenciado (nível 2), definido (nível 3), prevenção de defeitos (nível 4) e automação e otimização (nível 5). Cada um desses níveis é constituído por um conjunto de áreas de processo, sendo estas um conjunto de práticas que atendem a determinado objetivo (MPT, 2018).

\section{MATERIAIS E MÉTODO}

Para o desenvolvimento do presente trabalho, foram utilizadas diferentes bases de dados digitais, como: Google Scholar, IEEE Xplore, Biblioteca digital ACM, ScienceDirect e Portal Periódicos CAPES. O método aplicado consiste em uma revisão de literatura, a qual é caracterizada como uma busca de estudos primários sobre os modelos de maturidade de testes de software com o objetivo de identificar os estudos disponíveis e abordagens utilizadas nessa temática. As questões de pesquisa definidas foram "Quais modelos de maturidade de teste são apresentados na literatura?" e "Qual(is) modelo(s) de maturidade de teste é mais comumente empregado(s)?".

A estratégia de busca nas bases de dados consistiu em utilizar as frases/palavraschave: "Test process improvement" or "Software Test Maturity" AND "Software testing", considerando o período de 2008 a 2018. A query definida foi:

- Biblioteca digital ACM: Searched for recordAbstract: ("Test process improvement" OR "Software Test Maturity") AND (+"software test") [new search] [edit/save query] Total: 9;

- Google Scholar: Com as palavras "test process" ou "software test" or "test improvement model" and "software test". Total=24;

- IEEE Xplore: "Abstract":"Software Test Maturity") OR "Abstract":'Test Process
Improvement") AND "software test". Total=144.

Os critérios de exclusão considerados foram, primeiro, selecionar artigo de periódico com alto fator de impacto (Impact Factor) e de extrato superior (A1, A2, B1 e B2) na classificação Qualis Capes, na área de Ciências da Computação. Segundo, considerar trabalhos publicados em eventos na área de Engenharia de Software, Processo de Software e Qualidade de software. O último critério consistiu em selecionar artigos que tratam especificamente de modelos de maturidade de software com ênfase em testes.

\section{RESULTADOS E DISCUSSÃO}

A consulta nas diferentes bases digitais retornou um total de 177 trabalhos científicos. A partir da aplicação dos critérios de exclusão, um total de 17 artigos foi selecionado para discussão no presente trabalho.

Figura 7. Distribuição dos trabalhos em função do meio de divulgação.

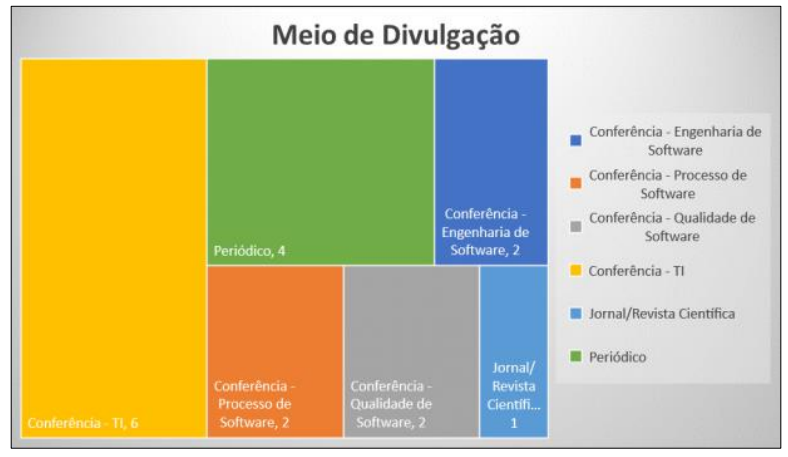

Fonte: Autor.

De acordo com a Figura 7, observa-se que o tema Modelos de Maturidade de Teste tem sido discutido em diferentes eventos científicos na área de TI (Tecnologia da Informação).

A Figura 8 mostra que $41 \%$ dos trabalhos analisados enfatizam a proposta de um novo modelo de maturidade de teste tendo como base um outro modelo de maturidade pré-existente. $\mathrm{Na}$ análise dos trabalhos, constatou-se que isto se deve a 
diversos motivos, tais como a falta de detalhes na avaliação, alto custo de implantação, complexidade do modelo e falta de aplicabilidade na área em específico.

Ainda com base na Figura 8, verificase que aproximadamente $30 \%$ dos trabalhos analisados consistem revisão sistemática da literatura e que foram publicados nos últimos dois anos.

Figura 8. Ênfase dos trabalhos analisados.

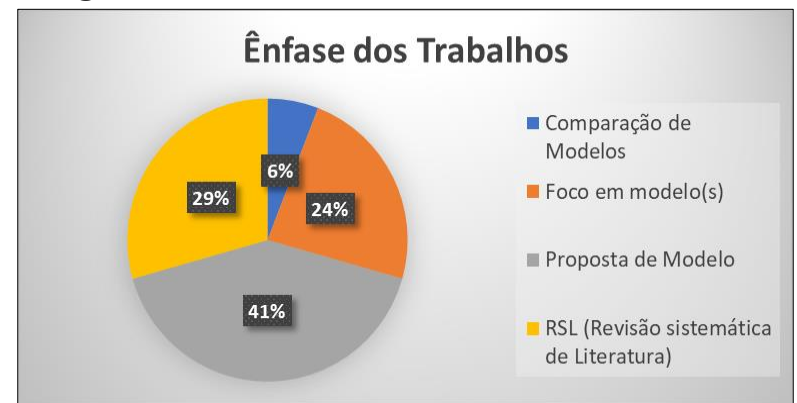

Fonte: Autor.

Um destes trabalhos é o de Afzal et al. (2016). Tais autores realizaram uma revisão sistemática da literatura para identificar as abordagens disponíveis de melhoria de processo de teste de software e identificaram um total de 18 abordagens. Porém, afirmam que muitas dessas abordagens são difíceis de serem aplicadas nas empresas devido à falta de informações como, por exemplo, instrumentos de avaliação. Afzal et al. (2016) concluem que cinco abordagens, em geral, são aplicáveis, quais sejam: TMM, TMMi, TPI, TPI- Next e Teste SPICE. No entanto, o TMMi e o TPI-Next são as abordagens e modelos mais populares e usados na área de teste de software.

Outros dois estudos nessa temática de revisão sistemática da literatura sobre o processo de avaliação de maturidade de teste e melhoria de processo de teste são os apresentados por (GAROUSI; FELDERER; HACALOĞLU, 2017; 2018). Tais autores revisaram um total de 181 trabalhos, dos quais 130 correspondem a trabalhos científicos, isto é, publicados em periódicos ou eventos científicos. O restante (total de 51) correspondem a trabalhos publicados em blogs, posts, artigos em sites e vídeos, os chamados trabalhos da literatura cinza. Garousi, Felderer e Hacaloğlu (2017; 2018) identificaram um total de 58 novos modelos de maturidade de teste e 117 modelos existentes, e confirmam os achados de Afzal et al. (2016), argumentando que o TMMi e seu antecessor, o TMM, e o TPI e seu sucessor, o TPI-Next, são os modelos de maturidade de teste mais populares. Garousi, Felderer e Hacaloğlu (2017; 2018) relatam que um total de 57 trabalhos apresentaram o TMMi e o TMM como modelo de avaliação ou modelo base para desenvolver o processo de avaliação, enquanto o TPI e o TPI-Next são encontrados em 18 trabalhos. Outros 28 trabalhos trazem outros modelos, tal como o SPICE e o TMap. Garousi, Felderer e Hacaloğlu (2017) argumentam, ainda, que os modelos e técnicas de maturidade em TMA/TPI atuais fornecem um suporte razoável para a indústria e para a comunidade científica da área.

Figura 9. Quantidade de trabalhos publicados no período (2008 a 2018).

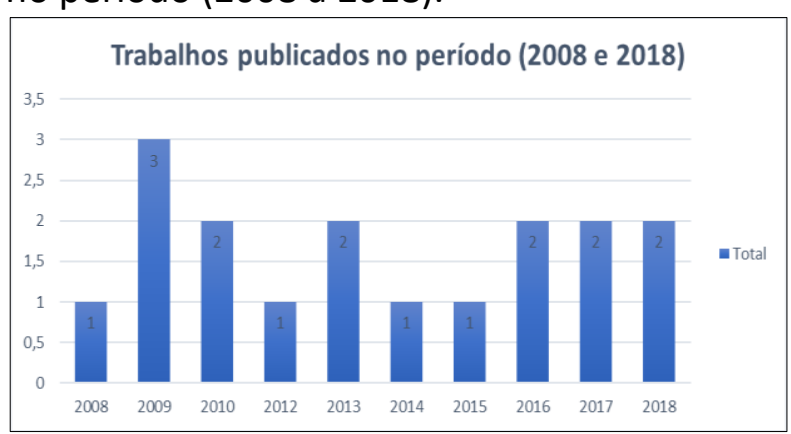

Fonte: Autor.

O presente trabalho mostra, com a Figura 9, que existe uma constância de publicações na área de TMA e TPI, ao longo da última década. A Figura 8 destaca que a maior parte dos trabalhos (41\%) enfatiza a proposta de um novo modelo baseado em outros modelos existentes. Em detalhe desses casos, a Tabela 1 mostra que a maioria dos novos modelos propostos utilizam o TMMi ou TPI como base para sua concepção. Tem-se que cinco (72\%) dos novos modelos propostos utilizam TMM e, três (43\%) utilizam o TPI como método base. A literatura mostra que isso decorre de 
fatores, tais como: alto custo dos modelos originais, dificuldade de adaptabilidade à outras áreas, complexidade dos modelos originais, falta de visão de retorno de investimento tendo em vista $\mathrm{o}$ alto investimento.

Ainda com relação à Tabela 1 , é possível notar que seis (86\%) dos modelos propostos são destinados a uma área em específico, o que leva a conclusão de que os modelos não são facilmente adaptáveis a qualquer área de produção de software.

Em Ryu, Ryu e Baik (2008), por exemplo, devido a necessidade de adoção de um modelo em um sistema de missão crítica, como o setor de armas, foi necessário acrescentar requisitos e participação de especialistas do setor. Desta forma, este e os demais modelos adaptados não necessitam de avaliadores oficiais e de toda a complexidade exigida dos modelos de origem.

Tabela 1. Trabalhos que focam em proposta de novos modelos de maturidade de teste.

\begin{tabular}{|c|c|c|c|c|}
\hline Descrição do trabalho & Modelo proposto & Sigla & Origem & Área \\
\hline $\begin{array}{l}\text { Inception of Software Validation and } \\
\text { Verification Practices within CMMI } \\
\text { Level } 2 \text { (Monteiro et al. 2009). }\end{array}$ & CMMi & CMMi & $\frac{\mathrm{CMMi} / \text { ISO- }}{\text { IEC } 29119}$ & $\begin{array}{c}\text { Softwares em } \\
\text { geral }\end{array}$ \\
\hline $\begin{array}{l}\text { A test process improvement model for } \\
\text { embedded software developments, in: } \\
\text { Quality Software (Garcia et al. } 2018 \text { ). }\end{array}$ & $\begin{array}{l}\text { Embedded Test } \\
\text { Process Improvement } \\
\text { Model }\end{array}$ & Emb-TPI & TMM/TPI & $\begin{array}{c}\text { Sistemas } \\
\text { embarcados }\end{array}$ \\
\hline $\begin{array}{l}\text { Adapting and Adjusting Test Process } \\
\text { Reflecting Characteristics of } \\
\text { Embedded Software and Industrial } \\
\text { Properties Based on Referential } \\
\text { Models (Lee, 2009) }\end{array}$ & \begin{tabular}{|l|} 
Test Process \\
Improvement for \\
Embedded software \\
and Industrial \\
characteristics
\end{tabular} & TPI-EI & TPI/TMM/TIF & $\begin{array}{c}\text { Sistemas } \\
\text { embarcados }\end{array}$ \\
\hline $\begin{array}{l}\text { A Strategic Test Process Improvement } \\
\text { Approach Using an Ontological } \\
\text { Description for MND-TMM (Ryu et } \\
\text { al. 2008). }\end{array}$ & $\begin{array}{l}\text { Ministry of National } \\
\text { Defense-Testing } \\
\text { Maturity Model }\end{array}$ & $\begin{array}{l}\text { MND- } \\
\text { TMM }\end{array}$ & TMM & $\begin{array}{l}\text { Sistemas de } \\
\text { armas } \\
\text { militares }\end{array}$ \\
\hline $\begin{array}{l}\text { Refining an Assessing Model for } \\
\text { Simplified TMM (Park et al. 2016) }\end{array}$ & $\begin{array}{l}\begin{array}{l}\text { Korean Test Maturity } \\
\text { Model }\end{array} \\
\end{array}$ & KTMM & TMM & PMEs \\
\hline $\begin{array}{l}\text { A Framework for Maturity } \\
\text { Assessment in Software Testing for } \\
\text { Small and Medium-Sized Enterprises } \\
\text { (Araújo et al. 2013) }\end{array}$ & $\begin{array}{l}\text { Framework for } \\
\text { Maturity Assessment } \\
\text { in Software Testing } \\
\text { for PMEs }\end{array}$ & - & TMM/TPI & PMES \\
\hline $\begin{array}{l}\text { Organizational testing management } \\
\text { maturity model for a software product } \\
\text { line Lamas et al. (2010). }\end{array}$ & $\begin{array}{l}\text { Organizational } \\
\text { Testing Management } \\
\text { Maturity Model }\end{array}$ & OTM3 & SPEM 2.0 & $\begin{array}{l}\text { Linha de } \\
\text { produtos de } \\
\text { software }\end{array}$ \\
\hline
\end{tabular}

\section{CONCLUSÃO}

Este trabalho identificou os modelos de maturidade de testes propostos para a melhoria do processo de teste de software. Conclui-se que existe muitos modelos de maturidade de teste propostos na literatura, sendo o TMMi e o TPI são os modelos mais utilizados a nível internacional. No entanto, cabe ressaltar que o MPT.br vem sendo também adotado pelas áreas de teste nas empresas de software no Brasil.
A revisão de literatura mostra que o grande volume de modelos propostos são adaptações de modelos existentes, sendo que o TMMi e (ou) TPI são, em muitos casos, os modelos adotados como referência. Observou-se que isso se deve, por exemplo, ao alto custo de implantação de um modelo tradicional, a dificuldade em adaptação às áreas de domínio específicas da empresa e a alta complexidade dos modelos tradicionais.

Recomenda-se que, em um trabalho futuro, seja realizada uma investigação sobre a satisfação das empresas brasileiras certificadas no modelo nacional de maior referência, o MPT.br.

\section{REFERÊNCIAS}

AFZAL, W. et al. Software test process improvement approaches: a systematic literature review and an industrial case study. Journal of Systems and Software, v.111, p.1-33, 2016. https://doi.org/10.1016/i.jss.2015.08.048

BRITTON, T. et al. Reversible Debugging Software, University of Cambridge, Judge Business School. Technical Report, 2013.

GARCIA， C.; MELÉNDEZ, K.; DÁVILA, A. Adoptability of Test Process Models: ISO/IEC 29119, TMMI y TPI from the small organization perspective. Computación $e$ informática. v. 7, n. 1. p. 45-64. 2018.

GAROUSI, V. et al. What we know about software test maturity and test process improvement. IEEE Software, v. 35, n. 1, p. 84-92, 2018. https://doi.org/10.1109/MS.2017.4541043

GAROUSI, V. et al. Software test maturity assessment and test process improvement: $A$ multivocal literature review. Information and Software Technology, v. 85, p. 16-42, 2017. https://doi.org/10.1016/i.infsof.2017.01.001

GAROUSI, V. et al. A survey of software engineering practices in Turkey. Journal of 
Systems and Software, v.108, p.148-177, 2015.

https://doi.org/10.1016/i.jss.2015.06.036

GAROUSI, V.; ZHI, J. A survey of software testing practices in Canada. Journal of Systems and Software, v. 86, p. 1354-1376, 2013.

https://doi.org/10.1016/i.jss.2012.12.051

GIS-MPSF. Guia de Implementação de Software - Parte 2: Nível F:2016. Fev. 2016. Disponível

em: http://www.softex.br/mpsbr/guias. Acesso em: maio 2018.

GD-CMMI. CMMI ${ }^{\circledR}$ for Development, Version 1.3. Nov. 2010. Disponível em: https://cmmiinstitute.com/resources/cmmidevelopment-version-13. Acesso em: jun. 2018.

GRINDAL, M.; OFFUTT, J.; MELLIN, J. On the testing maturity of software producing organizations. Testing: Academia \& Industry Conference Practice And Research Techniques, 2006.

MONTEIRO, P.; MACHADO, R. J.; KAZMAN, R. Inception of software validation and verification practices within CMMI Level 2. In: SOFTWARE ENGINEERING ADVANCES, ICSEA'09. INTERNATIONAL CONFERENCE ON IEEE, 4. Proceedings... 2009. p. 536-541.

MPT - SOFTEX. Melhoria do processo de teste brasileiro - MPT.Br. Disponível em http://mpt.org.br/mpt/mpt/. Acesso em: maio 2018.

NETO, O. N. B. et al. Metodologia para configuração de instrumentos para auxiliar iniciativas de melhoria do processo de teste de software multimodelos. Abakós, v. 2, n. 1, p.03-31, 2013. Disponível em: $<$ http://periodicos.pucminas.br/index.php/ab akos/article/view/P.2316-

9451.2013v2n1p3/5780>. Acesso em: jul. 2018.
RYU, H; RYU, D.; BAIK, J. A strategic test process improvement approach using an ontological description for MND-TMM. In: COMPUTER AND INFORMATION SCIENCE, ICIS 08., IEEE/ACIS INTERNATIONAL CONFERENCE ON. IEEE, 7. Proceedings... 2008. p.561-566.

https://doi.org/10.1109/ICIS.2008.78

SILVA, F. P.; CHAIM, M. L. O impacto da adoção o dos modelos de maturidade TMMi e MPT. Br na gestão de projetos de software. In: SIMPOSIO ARGENTINO DE INGENIERÍA DE SOFTWARE (ASSE)-JAIIO 46. Proceedings... Córdoba, 2017.

TMMI FOUNDATION. Software quality management. 2018. Disponível em: https://www.tmmi.org/.

VEENENDAAL, E. V.; WELLS, B. Test Maturity Model integration (TMMi): Guidelines for Test Process Improvement, Uitgeverij Tutein Nolthenius, 2012.

VISSER, B. et al. TPI ${ }^{\circledR}$ NEXT - Business Driven Test Process Improvement. UTN Publishers, 2013. p.293. 